\title{
Influence of social facilitation on energy intake among university students. a laboratory study
}

\begin{abstract}
Background: Eating with companions or eating in the presence of other people at mealtime has been shown can impact energy intake through social facilitation effect. Social facilitation may influence the adoption of undesirable energy intake and thus may affect an individual's weight status. Objective: This study assessed the effect of social facilitation on energy intake among participants in a laboratory setting. Method: Sixty-four participants (50 female and 14 male students) from a public university participated in three standardized lunch buffet sessions under three social facilitation situations: eat alone, eat with unfamiliar peers, and eat with familiar peers. The lunch session was scheduled one week apart. The participants were invited to serve themselves in three lunch buffets which consisted of white rice, chicken dish, spicy fermented soybean (tempeh) and stir-fried vegetable. Result: Repeated measures analysis of variance revealed statistically significant effect of social facilitation situations on energy intake among the students ( $p$-value< 0.05). On average, the total energy intake for lunch was found to increase when the students ate with familiar peers $(705 \pm 170 \mathrm{kcal})$ compared to eating in a group of unfamiliar peers $(587 \pm 129 \mathrm{kcal})$ and eating alone $545 \pm 119 \mathrm{kcal})$. Conclusion: This evidence supports the hypothesis that social facilitation affects students' energy intake where the impact is greater when eating in a group of familiar peers.
\end{abstract}

Keyword: Obesity; Energy intake; Social facilitation; University students; Social influence; Social context 